\title{
COASY protein-associated neurodegeneration
}

INSERM

\section{Source}

INSERM. (1999). Orphanet: an online rare disease and orphan drug data base. COASY protein-associated neurodegeneration. ORPHA:397725

COASY protein-associated neurodegeneration (COPAN) is a very rare, slowly progressive form of neurodegeneration with brain iron accumulation (NBIA) characterized by classic NBIA features. The clinical manifestations include early-onset spastic-dystonic paraparesis, oromandibular dystonia, dysarthria, parkinsonism, axonal neuropathy, progressive cognitive impairment, complex motor tics, and obsessive-compulsive disorder. 